Journal of

Accident and Emergency Medicine 1995 12, 273-275

\title{
Orbital blow-out fractures: long-term visual outcome of associated ocular injuries
}

\author{
D.G.R. JAYAMANNE \& R.F. GILLIE \\ Department of Ophthalmology, Newcastle General Hospital, Newcastle upon Tyne, UK
}

\section{SUMMARY}

Severe ocular injuries are uncommon in patients with pure orbital blow-out fractures. Over a 3year period, 45 patients with radiologically proved blow-out fractures were reviewed by an ophthalmologist. Decrease in visual acuity was the main clinical finding accompanying significant eye injury. Although most eye injuries were transient, early ophthalmic assessment is recommended to exclude potentially serious complications such as traumatic optic neuropathy. Troublesome diplopia may delay rapid rehabilitation.

Key words: diplopia, eye injuries, orbital blowout fractures, visual acuity

\section{INTRODUCTION}

Facial fractures are commonly associated with injury to the eye and adnexa, which may result in a loss of vision. Some injuries appear minimal, but mask severe ocular dysfunction, which may be missed by a non-ophthalmologist. All patients with an orbital blow-out fracture with associated reduction in visual acuity should be reviewed by an ophthalmologist. ${ }^{1}$ As ocular dysfunction may cause permanent visual disability, treatable defects such as retinal tears and holes should be identified early and treated. Estimates of the incidence of ocular disorder after mid-facial fractures vary from 2.7 to $67 \% .^{1,2}$

Converse and Smith were the first to describe the classical 'blow-out' fracture of the orbital floor without associated fracture of the orbital rim. ${ }^{3}$ The orbital blow-out fracture is characterized by depression of the floor of the orbit, often with herniation of the orbital contents into the maxillary antrum. The incidence of severe ocular disorder after orbital blow-out fractures is reported to be $16.7 \% .^{4}$ Previous reports have not assessed the long-term visual outcome of patients with orbital blow-out fractures.
This retrospective study assesssed the incidence of ocular injuries and visual outcome as assessed by ophthalmologists after orbital blowout fractures.

\section{SUBJECTS AND METHODS}

This study was based on the retrospective analysis of $\mathbf{4 5}$ patients who sustained radiologically proved pure orbital blow-out fractures. All patients with radiologically proved blow-out fractures who presented to the Newcastle General Hospital eye casualty department over a 3-year period were included in the study (January 1991 to January 1994). In addition, a consultant ophthalmologist with a special interest in orbital injuries assessed patients with orbital blow-out fractures who initially presented to the oral and maxillofacial department. These patients are also reported in this paper.

Details obtained were patient age, sex, mean follow-up time, history of injury, visual acuity at presentation, visual acuity at final outpatient appointment, presence of diplopia, range of ocular movement (clinical and Lees screen recordings), presence of infraorbital nerve anaesthesia, degree of enophthalmos or proptosis, evidence of surgical emphysema, recording of anterior chamber and posterior pole examination, surgical procedures performed and the outcome of surgery in terms of improvement of diplopia and cosmetic appearance. All patients were examined by a member of the ophthalmology department.

The final visual outcome of patients who sustained orbital blow-out fractures is reported. The final visual outcome was defined as the corrected visual acuity (spectacles or pin-hole) recorded at the last attendance of the patient. For patients who failed to return for follow-up, the final visual acuity was taken as the visual acuity recorded at the last clinic appointment. In addition, 
D.G.R. Jayamanne

\& R.F. Gillie the incidence of ocular coimplications after orbital blow-out fractures is reported.

\section{RESULTS}

The study included 45 patients with confirmed pure orbital blow-out fractures on radiological investigation. Six patients were diagnosed as having medial wall blow-out fractures in addition to orbital floor fractures.

The ages of the patients ranged from 12 to 69 years. A total of $40(89 \%)$ patients was men. The major cause of orbital blow-out fractures was assaults (73\%).

Visual acuity at presentation in the study group was greater than 6/9 in 40 (90\%) patients, increasing to $43(96 \%)$ patients at discharge. One patient had a visual acuity between $6 / 24$ and $6 / 60$ at discharge, which was due to a traumatic optic neuropathy. One patient had poor vision before the blunt injury (amblyopic eye). No patient had a visual acuity less than $6 / 60$ at discharge.

Motility disorders in association with blow-out fractures were common, with 38 (84\%) patients having some limitation of ocular movement in one or more directions of gaze. Six of these patients reported diplopia in the primary position of gaze.

Six $(13 \%)$ patients underwent early orbital floor repairs (within 14 days of the injury). All six patients reported diplopia in the primary position before surgery and after surgery three patients reported persistent diplopia, these three patients required extraocular muscle surgery for troublesome diplopia within 9 months of the initial orbital floor repairs. Interestingly, the remaining patients who reported diplopia on presentation had complete resolution of symptoms without surgical intervention.

Enophthalmos after blow-out fractures occurred in $62 \%$ of the patients. None of these patients underwent surgical exploration for gross enophthalmos.

Infraorbital nerve anaesthesia was present in nine $(20 \%)$ patients; in all instances it was transient.

Only a small minority of patients with a pure orbital blow-out fracture showed any abnormal anterior chamber or posterior pole findings. Three patients had microscopic hyphaema on slit lamp examination. Posterior pole examination showed three patients with small areas of retinal commotio and one patient with a macula sparing choroidal rupture. During the study period no patient sustained a retinal detachment or dialysis. All other complications noted are listed in Table 1.
Table 1. Associated ocular injuries with orbital blow-out fractures

\begin{tabular}{lc} 
Enophthalmos & 28 \\
Conjunctival injury & 3 \\
Hyphaema & 3 \\
Commotio retinae & 3 \\
Traumatic mydriasis & 2 \\
Corneal abrasion & 1 \\
Traumatic optic neuropathy & 1 \\
Choroidal rupture & 1 \\
Lens subluxation & 0 \\
Retinal detachment & 0 \\
Globe rupture & 0 \\
\hline
\end{tabular}

Total number of orbital blow-out fractures 45

\section{DISCUSSION}

In this study sever ocular complications in the orbital blow-out group were rare. In keeping with the low incidence of ocular complications, the final visual outcome of patients with pure orbital blowout fractures was excellent, with $43(96 \%)$ patients having a visual acuity greater than $6 / 9$ at discharge. Although previous studies have reported that many ocular injuries after orbital fractures are transient, ${ }^{4,5}$ long-term visual outcomes in terms of visual acuity have been poorly documented.

Non-surgical management of orbital blow-out fractures reported previously show the resolution of troublesome diplopia in nearly all patients. ${ }^{6}$ In this study all patients with blow-out fractures managed conservatively had complete resolution of diplopia without extraocular muscle surgery. Extraocular muscle surgery was required for persistent diplopia in three patients who underwent early orbital floor repairs. Owing to the small number of patients undergoing floor repairs, it is difficult to conclude whether the persistent diplopia was secondary to the severity of the initial injury or the result of scaring caused by the surgical repair. In our series all patients who underwent early surgical repairs of the orbital floor fractures had diplopia in the primary position; diplopia in the primary position of gaze is believed to be caused by more significant injuries. ${ }^{7}$

Enophthalmos occurred in $28(62 \%)$ patients in this study. The pathogenesis of enophthalmos is controversial. Enlargement of the bony orbit and a change in the soft tissue shape and position may be responsible. Fat atrophy appears to be less important. ${ }^{8}$ Surgical exploration is recommended for disfiguring enophthalmos. Fracture of the zygoma is the most common cause of late enophthalmos; 9 
Orbital blow-out fractures late enophthalmos in true blow-out fractures does occur, but the cause is most commonly the concomitant medial wall fracture. ${ }^{9}$ Medial wall fractures may cause restricted or painful abduction and orbital emphysema in addition to gross enophthalmos. In our study, no patient underwent surgical exploration for enophthalmos.

Fortunately, most of the ocular injuries were transient and of no permanent consequence. However, of particular importance was the patient with traumatic optic neuropathy, which is an uncommon, but potentially serious, complication. Optic nerve contusion or compression can result in a total loss of vision in a healthy looking eye. Haematoma, ischaemia or direct bone fragment penetration of the optic nerve may be the cause. ${ }^{10}$ Traumatic optic neuropathy is an impact injury to the optic nerve; the injury may be permanent or temporary." The incidence of the injury varies from 0.5 to $5 \%$ of patients with closed head trauma. Visual loss is usually instantaneous. Visual acuity in patients with indirect optic nerve trauma is usually significantly reduced. ${ }^{11}$ In patients with unilateral traumatic optic neuropathy, an afferent pupillary defect may be present. Traumatic optic neuropathy is one of the true ophthalmic emergencies. The window of opportunity for intervention in the treatment of intracanalicular optic nerve injuries may be less than 8 hours. ${ }^{11}$ Treatment with immediate decompression of the optic nerve and high doses of corticosteroids remains controversial. ${ }^{12}$ The initial goal in treating traumatic optic neuropathy is earty recognition. Performing a canthotomy, cantholysis if the globe is tense, and draining of subperiosteal haematoma if present is important.

Intravenous methylprednisolone is usually tried next, and if the vision improves with methylprednisolone, the patient is treated with steroids by mouth. A retrospective study reported moderate success using corticosteroids in the management of traumatic optic neuropathy. ${ }^{13}$ A non-consecutive retrospective report of patients treated with transethmoidal-sphenoidal optic canal decompression demonstrated that surgical intervention was helpful in 11 of 14 patients. ${ }^{14}$ It is higly probable that successful treatment will require both high doses of corticosteroids and surgical intervention. Definitive recommendations must await the results of prospective clinical trials. ${ }^{12}$

Orbital blow-out fractures are not ophthalmological emergency, ${ }^{15}$ and immediate surgical repair is seldom indicated. The time for an eye examination is at the initial assessment of the patient's trauma. Although the incidence of serious ocular injuries are low, it is suggested that all patients sustaining blow-out fractures with an associated reduction in visual acuity should be reviewed at an ophthalmology department at the earliest possible occasion after the injury so that ophthalmic assessment can be documented early and appropriate treatment initiated.

\section{REFERENCES}

1. Holt J.E., Holt G.R. \& Blodgett J.M. (1983) Ocular injuries sustained during blunt facial trauma. Ophthalmology 90, 14.

2. Luce E.A., Tubb T.D. \& Moore A.M. (1979) Review of 1000 major facial fractures and associated injuries. Plastic and Reconstructive Surgery 63, 26.

3. Converse J.M. \& Smith B. (1957) Enophthalmos and diplopia in fractures of the orbital floor. British Journal of Plastic Surgery 9, 265.

4. Al-Qurainy A., Stassen L.F.A., Dutton G.N., Moos K.F. \& El-Attar A. (1991) The characteristics of midfacial fractures and the association with ocular injury: a prospective study. British Journal of Oral and Maxillofacial Surgery 29, 301.

5. Patton D. \& Emery J.M. Injuries of the eye, the lids, and the orbit. In: Ballinger, W.F. (ed.) Management of Trauma 2nd edn, pp. 219-254. Saunders, Philadelphia.

6. Putterman A.M., Stevens T. \& Urist M.J. (1974) Non surgical management of blow-out fractures of the orbital floor. American Journal of Ophthalmology 77, 232.

7. Al-Quarainy A., Stassen F.A., Dutton G.N., Moos K.F. \& El-Attar A. (1988) Diplopia following midfacial fractures. British Journal of Oral and Maxillofacial Surgery 29, 302.

8. Manson P.N., Grivas A. \& Rosenbaum A. (1986) Studies on enophthalmos: ii, the measurement of orbital injuries and their treatment by quantitative computed tomography. Plastic and Reconstructive Surgery 77, 203.

9. Pearl R.M. (1992) Treatment of enophthalmos. Clinics in Plastic Surgery 19, 99.

10. Ketchum L.D., Ferris B. \& Masters F.W. (1976) Blindness without direct injury to the globe: a complication of facial fractures. Plastic and Reconstructive Surgery 58, 187.

11. Steinsapir K.D. \& Goldberg R.A. (1994) Traumatic optic neuropathy. Survey of Ophthalmology 38, 487.

12. Miller N.R. (1990) The management of traumatic optic neuropathy [editorial]. Archives of Ophthalmology 108, 1086.

13. Spoor T.C., Hartel W.C., Lensink D.B. \& Wilkingson M.J. (1990) American Journal of Ophthalmology 110, 665.

14. Joseph M.P., Lessell S., Rizzo J. \& Momose K.J. (1990) Extracranial optic nerve decompression for traumatic neuropathy. Archives of Ophthalmology 108, 1091.

15. Catone G.A., Marrissette M.P. \& Carlson E.R. (1988) A retrospective study of untreated orbital blow-out fractures. Joumal of Oral and Maxillofacial Surgery 46, 1033-1037. 